\title{
The importance of the mean platelet volume in the diagnosis of supraventricular tachycardia
}

\author{
Ocak T1, Erdem A², Duran A1, Tekelioglu U², Öztürk S², Ayhan S², Özlü FM², Yazici M²
}

1. Department of Emergency Medicine, Abant Izzet Baysal University School of Medicine, Bolu, Turkey.

2. Department of Cardiology, Abant Izzet Baysal University School of Medicine, Bolu, Turkey.

3. Department of Anesthesiology and Reanimation, AbantIzzet Baysal University School of Medicine, Bolu, Turkey

\begin{abstract}
Background: This retrospective study aimed to investigate the diagnostic relation between the mean platelet volume (MPV) and supraventricular tachyarrhythmia (SVT) in patient with documented atrial tachyarrhythmia in the emergency department (ED).

Methods: Two study groups were compared; a SVT group with arrive at the ED with documented SVT $(n=122)$ and 100 healthy adult without any palpitation symptom, arrhythmic disease, and with normal physical examination results that were brought for checkups to the cardiology polyclinic were classified as control group. Blood samples were obtained from all patients for determining the hematologic counts and MPV during first hour in ED period.

Results: In terms of the focus of the study, hemoglobin, neutrophil count, mean cell volume (MCV), red cell distribution width (RDW), platelet, white blood cell (WBC), and lymphocyte counts were similar in both group ( $>>0.05)$. MPV in the SVT group was significantly higher than in the control group $(9.12 \pm 1.22 \mathrm{flvs} 8.64 \pm 0.89 \mathrm{fl}, \mathrm{p}<0.001)$. Multivariate logistic regression analysis showed that just MPV was independent predictor of SVT in patients with palpitation in ED (odds ratio [OR] 8.497, 95\% confidence interval (6.181 to 12.325$), \mathrm{p}=0.012$ ).

Conclusions: The present study described that MPV is helpful parameter for the diagnosis of SVT in emergency department, for the first time in the literature.

Keywords: Mean Platelet Volume, Inflammation, Palpitation, Supraventricular Tachycardia, Diagnostic

African Health Sciences 2013; 13(3): 590 - 594 http://dx.doi.org/10.4314/ahs.v13i3.10
\end{abstract}

\section{Introduction}

Palpitation is one of the most common symptoms of patients presenting to the emergency department (ED) and found in up to $10 \%$ of the patients who applied to $\mathrm{ED}^{1}$. Many patients with palpitation arrive at the ED with or without documented supraventricular tachyarrhythmia (SVT). The diagnosis of palpitation can be difficult in the ED and the waiting time for a first appointment with an arrhythmia clinic can be very long. Previous studies have shown that inflammation is sufficient to facilitate the initiation of SVT, and recent evidence has demonstrated that an elevated inflammation markers may predict atrial tachycardia's in some patients ${ }^{1-3}$.

The mean platelet volume (MPV) is potentially one of the most important biomarkers of platelet activity and calculated by automatic blood count equipment during routine blood counts ${ }^{4}$. MPV

\footnotetext{
*Corresponding author:

Alim Erdem MD

Assistant Professor in Department of Cardiology AbantIzzet Baysal University Medical Faculty

14280 Golkoy - Bolu, Turkey

E-mail: cardiology14@gmail.com
}

is a marker of platelet function and is positively associated with indicators of platelet activity. An increased MPV is an indicator of larger and more reactive platelets ${ }^{5}$. Previous studies showed that increased mean platelet volume (MPV) is closely correlated with inflammation and to reflect inflammatory burden in different condition ${ }^{6-8}$. In our knowledge, this is the first study in the literature about the relation between SVT and MPV.

In this study, we aimed to investigate the relation between MPV and SVT in patient with documented atrial tachyarrhythmia in ED.

\section{Methods}

This retrospective study was approved by our Institutional Ethics Committee of Abant Izzet Baysal University Medical School. Patient data were obtained from the computer record of our hospital and analyzed. This study was performed in the ED of Abant Izzet Baysal University, School of Medicine Hospital between September 2011 and December 2012. 
During the study period, 248 consecutive patients with palpitation were treated in the ED. A total of 126 patients were excluded from this study due to missing information and exclusion criteria. We included 122 patients who arrive at the ED with documented SVT and 100 healthy adult without any palpitation symptom, arrhythmic disease, and with normal physical examination results that were brought for checkups to the cardiology polyclinic were classified as control group.

Patients were excluded by the following criteria: to have elevated serologic markers of inflammation independent of the association with their arrhythmia: patients with a history of a myocardial infarction or elevated troponin levels within the previous 3 months, major trauma or surgery within the previous 3 months, any chronic inflammatory disease (including chronic rheumatologic diseases requiring immunosuppressive agents), chronic infectious diseases requiring treatment, any active malignancy, any acute rheumatologic or infectious disease (including symptoms of a common upper respiratory tract infection), or any other condition that would be expected to cause a fever, elevated white blood cell count, or elevated erythrocyte sedimentation rate. Those who might have been unable to produce elevated serologic markers were also excluded: patients on immunosuppressive therapy (e.g. steroids), and those with leukopenia of any etiology. Patients with congenital heart disease (corrected or not) were also excluded.

Age, gender, leukocyte and thrombocyte counts at the time of hospital admission, MPV values, and ECG were recorded. The MPV was determined on arrival at the ED through the brachial vein, collected into tubes containing ethylenediaminetetraacetic acid (EDTA), and processed within 1 hour after venipuncture. For the measurements of the hematologic counts and MPV, samples were analyzed within 20 min after collection with an automated flow counter (Beckman Coulter LH 750, USA).

The results are presented as mean $\pm \mathrm{SD}$ or frequency (percentage) as appropriate. A \% test or Fisher exact test, as appropriate, was used for the statistical analysis of categorical variables. Continuous variables were compared using Mann-Whitney $\mathrm{U}$ test. One-way analysis of variance (ANOVA) with Fisher LSD comparisons was used to compare more than two parametric variables. The variables with univariate comparisons of $\mathrm{p}<0.05$ between SVT and control group were eligible for inclusion in an entry selection multiple logistic regression model, which identified the clinical factors in the ED that were potential predictors of SVT. To determine the accuracy and respective best cut-off values of MPV for predicting SVT, the receiver operating characteristic (ROC) curves and their corresponding areas under the curve were used. A p value of $<0.05$ was considered statistically significant. A common statistical package (SPSS 17.0 for Windows; SPSS, Chicago, Illinois, USA) was used to perform all statistical tests.

\section{Result}

During the 16 month period of the study, 248 consecutive patients visited the ED due to palpitation. Of those, 126 were excluded. Thus, 122 patients (46 men and 76 women) were eligible for enrolment in the final analysis. The demographic and clinical characteristics of the study patients are depicted in table 1. In terms of the focus of the study, hemoglobin, neutrophil count, mean cell volume (MCV), red cell distribution width (RDW), platelet, white blood cell (WBC), and lymphocyte counts were similar in both groups (table 1). MPV in the SVT group was significantly higher than in the control group (9.12 \pm 1.22 flvs $8.64 \pm 0.89 \mathrm{fl}, \mathrm{p}<0.001$; figure 1).

In order to assess the potential independent predictors of SVT in patients with palpitation, multiple logistical regression analysis was performed and included data yielding significant differences $(p<0.05)$ between the SVT group and the control group individually.

After adjusting for demographic and all hematologic factors (age, gender, smoking, body mass index, white blood cell count, hemoglobin, hematocrit, neutrophil count, MCV, RDW, MPV, leucocyte count and platelet count), it was found that just MPV showed significance in a binary logistical regression model (odds ratio [OR] 8.497, 95\% confidence interval (6.181 to 12.325), $\mathrm{p}=0.012$ ).

The ROC curve analysis of MPV when predicting SVT in patients with palpitation was constructed and the areas under the curve was found to be 0.583 ( $95 \%$ CI 0.506 to $0.660, p=0.036$; figure 2). The best cut-off values for MPV when predicting SVT in patients with palpitation presenting at the ED were 7.95 fl (sensitivity 88.2\%; specificity 32.3\%) 
Table 1: Baseline demographic characteristics and hematologic parameters of the study population

\begin{tabular}{llll}
\hline & SVT (n=126) & Control (n=100) & p value \\
\hline Age (years) & $32.9 \pm 8.4$ & $32.2 \pm 8.6$ & 0.231 \\
Gender (male/female) & $34 / 92$ & $30 / 70$ & 0.618 \\
Smoking, n (\%) & $37(29.3 \%)$ & $22(22.0 \%)$ & 0.097 \\
Creatinine (mg/dl) & $1.01 \pm 0.4$ & $0.89 \pm 0.22$ & 0.802 \\
Hemoglobin, g/dL & $13.20 \pm 1.75$ & $13.32 \pm 1.63$ & 0.605 \\
Hematocrite & $39.45 \pm 1.74$ & $39.84 \pm 4.37$ & 0.527 \\
MCV, fL & $82.9 \pm 8.6$ & $81.8 \pm 8.3$ & 0.549 \\
RDW (\%) & $16.58 \pm 0.68$ & $16.54 \pm 0.50$ & 0.612 \\
Platelet count, K/uL & $239.01 \pm 78.16$ & $253.64 \pm 64.03$ & 0.527 \\
MPV, fl & $9.11 \pm 1.21$ & $8.64 \pm$ & 0.89 \\
WBC count & $7.62 \pm 1.84$ & $7.66 \pm 1.90$ & 0.901 \\
Lymphocyte, K/uL & $2.1[1.4]$ & $2.2[1.2]$ & 0.324 \\
Neutrophil, x10 9 & $6.5[1.5]$ & $6.3[1.5]$ & 0.231 \\
\hline
\end{tabular}

Data are shown as mean \pm SD, median [interquartile range] *Student's t-test and Mann Whitney U test were used. MCV, mean corpuscular volume; MPV, mean platelet volume; RDW, red cell distrubition width; WBC, white blood cell.

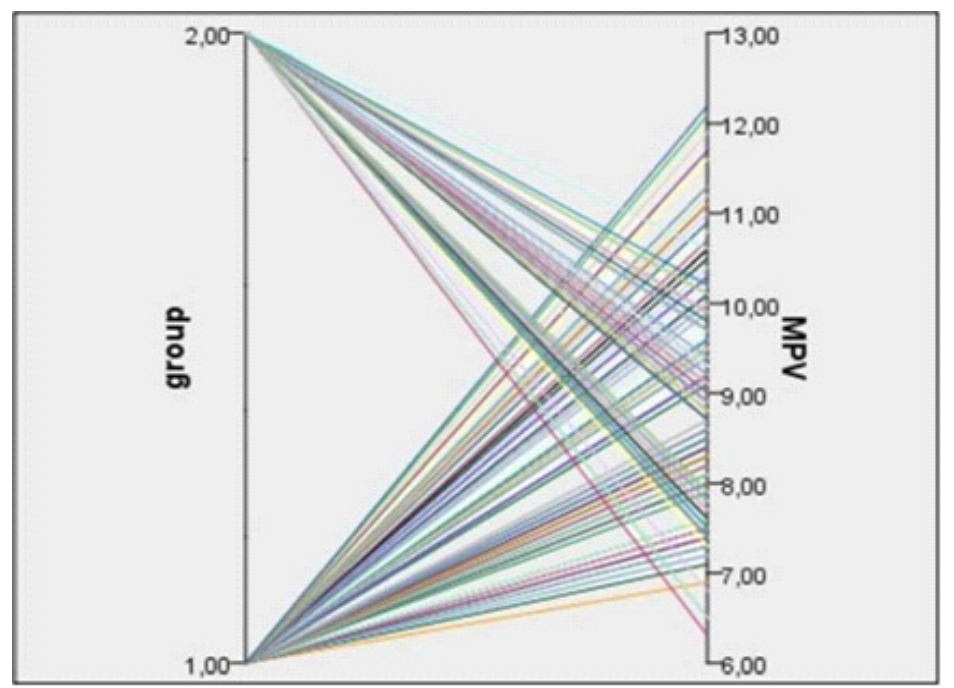

Figure 1: The comparison of MPV level in two groups

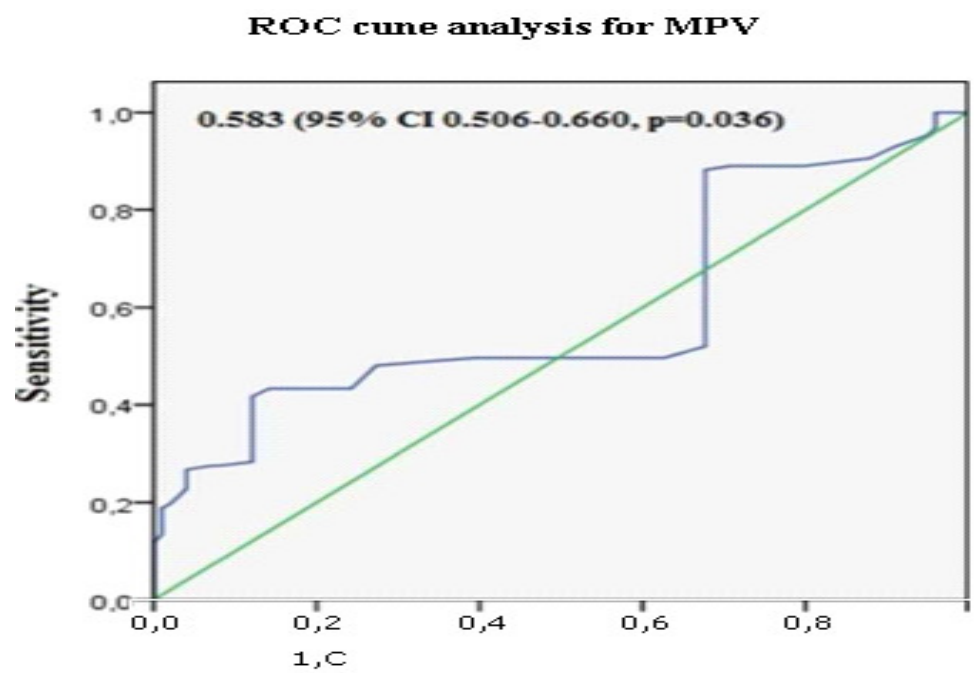

Figure 2: ROC curve analysis for MPV 


\section{Discussion}

This study demonstrated that MPV was significantly higher in patients with documented SVT in ED than in control subject. In addition, we showed that MPV was an independent predictor of SVT. To the best of our knowledge, this the first study that evaluate the relationship between MPV and SVT in the literature.

Platelets are blood elements with various densities and sizes. Platelets have important pathophysiological roles, including inflammation and coagulation ${ }^{9}$. Previous studies have been shown that platelet volume, when measured as MPV, is a marker of platelet function and is positively associated with indicators of platelet activity ${ }^{10}$. In conclusion, an increased MPV is shown more reactive platelets. Previous studies showed that increased in MPV is closely correlated with inflammation and to reflect inflammatory burden in different condition ${ }^{6-8}$. MPV is a parameter easily calculated by automatic blood count equipment during routine blood counts in all ED services. However, most of time MPV is not generally taken into consideration by physicians.

SVT's are the most common encountered diseases seen in emergency department. Many patients with this symptom arrive at the ED with or without documented SVT. The diagnosis of SVT can be difficult in the ED and the waiting time for a first appointment with an arrhythmia clinic can be very long. A detailed history, physical examination, and electrocardiography (ECG) are of limited value in the differential diagnosis, because most rhythm disturbances are of a paroxysmal nature ${ }^{11}$. Despite this approach, in many cases the etiology of palpitation remains unclear in the ED. The literature contains only limited data from small-scale studies assessing the diagnostic values of biomarkers in patients presenting to the ED with SVT ${ }^{12-14}$. Previous studies concluded that inflammation may play an important role in SVT ${ }^{15,16}$. Chung et all concluded that an elevated inflammation markers (specially Creactive protein) may predict atrial tachycardia's in some patients. They found that CRP was elevated in patients with lone atrial arrhythmias in the absence of structural heart disease when compared with the control subjects. Also, they suggested that elevated CRP may be related to the "burden" of $\mathrm{AF}^{1}$. Fustaci et al. demonstrated that marked inflammatory infiltrates in the atrial biopsies of patients with lone AF ${ }^{17}$. Additionally, previous studies concluded a decrease in inflammation after cure of atrial arrhythmias ${ }^{15,18}$. The results of all these previous studies suggested that atrial tachyarrhythmia's may be responsible for or at least stimulate a systemic inflammatory process. We found in our study as a similar to previous results that MPV was significantly higher in patients with documented SVT in ED than in control subject. Also, this study concluded that MPV was an independent predictor of SVT. We hypothesis that all of these relation can be helpful in ED for the diagnosis of SVT.

\section{Limitation}

The main limitation of our study was the small sample size. A small sample size can result in a low statistical power for equivalency testing, leading to false-negative results.

However, establishing a study group without comorbidities (e.g. diabetes mellitus, hypertension, and cardiovascular or renal disorders) is difficult. Second, because of the retrospective nature of data collection, echocardiographic parameters were not obtained concomitantly with blood sampling. Also, we were unable to evaluate some factors, such as hormone replacement therapy and some drugs, which have been effected to the MPV, because data on these variables were not collected.

\section{Conclusion}

The present report is the first study evaluating the relationship between MPV and SVT in ED. In brief, the present study described that MPV is helpful parameter for the diagnosis of SVT in emergency department, for the first time in the literature.

\section{References}

1. Chung MK, Martin DO, Sprecher D, et al. Creactive protein elevation in patients with atrial arrhythmias: inflammatory mechanisms and persistence of atrial fibrillation. Circulation. 2001;104:2886-2891.

2. Marcus GM, Smith LM, Glidden DV, et al. Markers of inflammation before and after curative ablation of atrial flutter. Heart Rhythm. 2008 Feb;5(2):215-21.

3. Psychari SN, Apostolou TS, Sinos L, et al. Relation of elevated C-reactive protein and interleukin-6 levels to left atrial size and duration of episodes in patients with atrial fibrillation. $\mathrm{Am}$ J Cardiol. 2005;95:764-767.

4. Gawaz M, Langer H, May AE. Platelets in inflammation and atherogenesis. J Clin Invest 2005; 115: 3378-3384. 
5. Bilici S, Sekmenli T, Göksu M, Melek M, Avci $\mathrm{V}$. Mean platelet volume in diagnosis of acute appendicitis in children. Afr Health Sci. 2011 Sep;11(3):427-32.

6. Milovanovic $M$, Nilsson E, Järemo P. Relationships between platelets and inflammatory markers in rheumatoid arthritis. ClinChimActa. 2004; 343: 237-240.

7. Yazici S, Yazici M, Erer B, et al. The platelet indices in patients with rheumatoid arthritis: mean platelet volume reflects disease activity. Platelets. 2010; 21: 122-125.

8. Kisacik B, Tufan A, Kalyoncu U, et al. Mean platelet volume (MPV) as an inflammatory marker in ankylosing spondylitis and rheumatoid arthritis. Joint Bone Spine. 2008; 75: 291-294.

9. Kamath S, Blann AD, Lip GY. Platelets and atrial fibrillation. Eur Heart J. 2001 Dec;22(24):223342. Review.

10. Gasparyan AY, Ayvazyan L, Mikhailidis DP, et al. Mean platelet volume: a link between thrombosis and inflammation? Curr Pharm Des. 2011;17(1):47-58. Review.

11. Slovis CM, Kudenchuk PJ, Wayne MA, et al. Prehospital management of acute tachyarrhythmias. Prehosp Emerg Care. 2003 JanMar;7(1):2-12. Review.

12. Nilsson G, Pettersson A, Hedner J, et al. Increased plasma levels of atrial natriuretic peptide (ANP) in patients with paroxysmal supraventricular tachyarrhythmias. Acta Med Scand. 1987;221(1):15-21.
13. Zellweger MJ, Schaer BA, Cron TA, et al. Elevated troponin levels in absence of coronary artery disease after supraventricular tachycardia. Swiss Med Wkly. 2003;133:439-441.

14. Redfearna DP, Ratibb K, Marshalla HJ, et al. Supraventricular tachycardia promotes release of troponin I in patients with normal coronary arteries. International Journal of Cardiology.2005;102:521-522.

15. Marcus GM, Smith LM, Glidden DV, et al. Markers of inflammation before and after curative ablation of atrial flutter. Heart Rhythm. 2008 Feb;5(2):215-21.

16. Psychari SN, Apostolou TS, Sinos L, et al. Relation of elevated C-reactive protein and interleukin-6 levels to left atrial size and duration of episodes in patients with atrial fibrillation. $A m$ J Cardiol. 2005;95:764-767.)

17. Frustaci A, Chimenti C, Bellocci F, et al. Histological substrate of atrial biopsies in patients with lone atrial fibrillation. Circulation. 1997; 96: 1180-1184.

18. Kallergis EM, Manios EG, Kanoupakis EM et al. The role of the post-cardioversion time course of hs-CRP levels in clarifying the relationship between inflammation and persistence of atrial fibrillation. Heart. 2008;94:200-204. 